\title{
THE TENSOR PRODUCT OF DISTRIBUTIVE LATTICES: STRUCTURAL RESULTS
}

\author{
by ANDREW M. BELL, MICHAEL R. BROWN and GRANT A. FRASER $\dagger$
}

(Received 14th October 1983)

The tensor product $A \otimes B$ of the distributive lattices $A$ and $B$ was first investigated by Fraser in [4] and [5]. In this paper, we present some results relevant to the structure and construction of this tensor product. In particular, we establish a sufficient condition for join-irreducibility in the tensor product and show that this condition characterizes join-irreducibility in the case that $A$ and $B$ satisfy the descending chain condition. We also show that if $A$ and $B$ satisfy the descending chain condition then so does $A \otimes B$; this insures the compact generation of $A \otimes B$ by its join-irreducibles. We conclude with some examples and applications of our results to the tensor product of finite distributive lattices.

\section{Definitions and preliminaries}

Details of the existence, uniqueness, and construction of the tensor product $A \otimes B$ of the distributive lattices $A$ and $B$ appear in [4] and [5]. We summarize these results briefly.

Definition 1.1. Let $A, B$ and $C$ be distributive lattices. A function $f: A \times B \rightarrow C$ is called a bihomomorphism if the functions $g_{a}: B \rightarrow C$ defined by $g_{a}(b)=f(a, b)$ and $h_{b}: A \rightarrow C$ defined by $h_{b}(a)=f(a, b)$ are lattice homomorphisms for each $a \in A$ and $b \in B$.

Definition 1.2. Let $A$ and $B$ be distributive lattices. A distributive lattice $C$ is a tensor product of $A$ and $B$ if there exists a canonical bihomomorphism $f: A \times B \rightarrow C$ such that $C$ is generated by $f(A \times B)$ and for any distributive lattice $D$ and any bihomomorphism $g: A \times B \rightarrow D$ there is a homomorphism $h: C \rightarrow D$ satisfying $g=h f$.

Theorem 1.3. Let $A$ and $B$ be distributive lattices. Then a tensor product of $A$ and $B$ exists and is unique up to isomorphism.

The tensor product of $A$ and $B$ is denoted by $A \otimes B$ and the image of an element $(a, b)$ of $A \times B$ under the canonical bihomomorphism $f: A \times B \rightarrow A \otimes B$ is written as

$\lceil$ This research was partially supported by National Science Foundation Grants SPI 78-27194 and SPI 79-26614. 
$a \otimes b$. In this notation, the tensor product $A \otimes B$ is the distributive lattice which is generated by the elements of the form $a \otimes b$, subject to the partial ordering relation on these generators which is inherited from the direct product and to the four bihomomorphic identities:

$$
\begin{gathered}
\left(a_{1}+a_{2}\right) \otimes b=\left(a_{1} \otimes b\right)+\left(a_{2} \otimes b\right) \\
\left(a_{1} a_{2}\right) \otimes b=\left(a_{1} \otimes b\right)\left(a_{2} \otimes b\right) \\
a \otimes\left(b_{1}+b_{2}\right)=\left(a \otimes b_{1}\right)+\left(a \otimes b_{2}\right)
\end{gathered}
$$

and

$$
a \otimes\left(b_{1} b_{2}\right)=\left(a \otimes b_{1}\right)\left(a \otimes b_{2}\right)
$$

for all $a, a_{1}, a_{2} \in A$ and all $b, b_{1}, b_{2} \in B$.

(The usual additive and multiplicative notation will be used to represent the join and meet operations in a lattice.)

\section{Prime filters and join-irreducibles in the tensor product}

For a distributive lattice $L$, let $S(L)$ be the set of prime filters in $L$, and let $S^{\prime}(L)=$ $S(L) \cup\{\phi, L\}$. Let 2 denote the two-element chain $\{0,1\}$ with $0<1$. The one-to-one correspondence between the elements $P$ of the set $S^{\prime}(L)$ and homomorphisms $h: L \rightarrow 2$ is well-known. The following characterization of $S^{\prime}(A \otimes B)$ was first obtained by Fraser in [4].

That there is a one-to-one correspondence between homomorphisms $h: A \otimes B \rightarrow C$ and bihomomorphisms $g: A \times B \rightarrow C$ follows from the definition of the tensor product. Thus there is a one-to-one correspondence between $P \in S^{\prime}(A \otimes B)$ and bihomomorphisms $g: A \times B \rightarrow 2$. This correspondence is such that $a \otimes b \in P$ if and only if $g(a, b)=1$.

Let $\mathscr{G}$ be the set of all bihomomorphisms $g: A \times B \rightarrow 2$. We define a partial order on $\mathscr{G}$ by setting $g_{1} \leqq g_{2}$ if and only if $g_{1}(a, b)=1$ implies $g_{2}(a, b)=1$ for all $a \in A, b \in B$. Now let $P, Q \in S^{\prime}(A \otimes B)$ and let $g_{P}, g_{Q}$ be the corresponding bihomomorphisms in $\mathscr{G}$. Then

$$
\begin{aligned}
g_{P} \leqq g_{Q} \Leftrightarrow & g_{P}(a, b)=1 \text { implies } g_{Q}(a, b)=1 \\
& \text { for all } a \in A, b \in B \\
\Leftrightarrow & a \otimes b \in P \text { implies } a \otimes b \in Q \\
& \text { for all } a \in A, b \in B \\
\Leftrightarrow & P \subseteq Q .
\end{aligned}
$$

This establishes that $S^{\prime}(A \otimes B)$ is order isomorphic to $\mathscr{G}$; the problem of characterizing $S^{\prime}(A \otimes B)$ then reduces to that of characterizing the bihomomorphisms $g: A \times B \rightarrow 2$. 
Now let $g: A \times B \rightarrow 2$ be a bihomomorphism and for each $x \in A$, let $F(x)=\{y \in B$ : $g(x, y)=1\}$. Then it follows from the bihomomorphic properties of $g$ that $F(x) \in S^{\prime}(B)$ and that for all $x_{1}, x_{2} \in A$,

$$
F\left(x_{1} x_{2}\right)=F\left(x_{1}\right) \cap F\left(x_{2}\right)
$$

and

$$
F\left(x_{1}+x_{2}\right)=F\left(x_{1}\right) \cup F\left(x_{2}\right) .
$$

The second identity establishes that the union of two members of the range of $F$ is again in the range of $F$. But the union of two filters is a filter only if one is contained in the other. Thus the range of $F$ is a chain and $F$ is a homomorphism from $A$ into a subchain of $S^{\prime}(B)$.

Conversely, if $F: A \rightarrow S^{\prime}(B)$ is such a homomorphism, define $g: A \times B \rightarrow 2$ such that $g(x, y)=1$ if and only if $y \in F(x)$. It is immediate that $g$ is a bihomomorphism in the set $\mathscr{G}$.

It is a straightforward verification that if $g_{1}$ and $g_{2}$ are distinct bihomomorphisms from $A \times B$ into 2, then the corresponding homomorphisms $F_{1}$ and $F_{2}$ from $A$ into $S^{\prime}(B)$ are also distinct.

Finally, let $\mathscr{H}$ be the set of all homomorphisms $F: A \rightarrow S^{\prime}(B)$ such that the range of $F$ is a subchain of $S^{\prime}(B)$. Let $\mathscr{H}$ be ordered by setting $F_{1} \leqq F_{2}$ if and only if $F_{1}(x) \subseteq F_{2}(x)$ for all $x \in A$. Then we have the following result.

Theorem 2.1. Let $A$ and $B$ be distributive lattices. Then $S^{\prime}(A \otimes B)$ is order isomorphic to the set $\mathscr{H}$ of all homomorphisms $F: A \rightarrow S^{\prime}(B)$ from $A$ into a subchain of $S^{\prime}(B)$.

Proof. Let $g_{1}, g_{2}$ be bihomomorphisms in $\mathscr{G}$ and let $F_{1}$ and $F_{2}$ be the corresponding homomorphisms in $\mathscr{H}$. Then

$$
\begin{aligned}
F_{1} \leqq F_{2} & \Leftrightarrow F_{1}(x) \subseteq F_{2}(x) \text { for all } x \in A \\
& \Leftrightarrow y \in F_{1}(x) \text { implies } y \in F_{2}(x) \text { for all } x \in A, y \in B \\
& \Leftrightarrow g_{1}(x, y)=1 \text { implies } g_{2}(x, y)=1 \text { for all } x \in A, y \in B \\
& \Leftrightarrow g_{1} \leqq g_{2} .
\end{aligned}
$$

The previously established order isomorphism of $\mathscr{G}$ and $S^{\prime}(A \otimes B)$ is then applicable and completes the proof.

This characterization of $S^{\prime}(A \otimes B)$ provides us with a first method for the construction of $A \otimes B$. It follows from Stone's representation theorem that for every distributive lattice $L$, the map $x \rightarrow x^{*}$, where $x^{*}=\{P \in S(L): x \in P\}$, determines an isomorphism between $L$ and a ring of upper-hereditary subsets of $S(L)$. (A subset $H$ of a partially ordered set $T$ is called upper-hereditary if for all $x, y \in T, x \in H$ and $x \leqq y$ implies $y \in H$. Lower-hereditary subsets are defined analogously.) 
Let $\mathscr{H}_{0}$ denote the set of all homomorphisms $F \in \mathscr{H}$ except the homomorphism $F$ defined by $F(x)=B$ for all $x \in A$. (This homomorphism corresponds to the bihomomorphism $g: A \times B \rightarrow 2$ which is such that $g(x, y)=1$ for all $x \in A$ and $y \in B$.)

Stone's theorem can be applied using the sets $(a \otimes b)^{*}=\{P \in S(A \otimes B): a \otimes b \in P\}$. Then $A \otimes B$ is isomorphic to the ring of subsets of $S(A \otimes B)$ generated by the sets $(a \otimes b)^{*}$. Since $(a \otimes b)^{*}$ is isomorphic to $\left\{F \in \mathscr{H}_{0}: b \in F(a)\right\}$, it is more convenient to use these subsets of $\mathscr{H}_{0}$ to generate a ring of sets isomorphic to $A \otimes B$.

We now have the necessary machinery at hand for an analysis of the join-irreducibles in $A \otimes B$. Recall that in any distributive lattice $L, x \in L$ is join-irreducible in $L$ if and only if the principal filter $[x]$ is prime in $L$. We begin by establishing a sufficient condition for join-irreducibility in the tensor product.

Theorem 2.2. Let $A$ and $B$ be distributive lattices and let $\left\{a_{i}\right\},\left\{b_{i}\right\}, 1 \leqq i \leqq n$, be sets of join-irreducibles in $A$ and $B$ which are such that $a_{1} \leqq \ldots \leqq a_{n}$ and $b_{1} \geqq \ldots \geqq b_{n}$. Then $\prod_{i=1}^{n}\left(a_{i} \otimes b_{i}\right)$ is join-irreducible in $A \otimes B$.

Proof. Let $f:\left\{a_{1}, \ldots, a_{n}\right\} \rightarrow S^{\prime}(B)$ be the mapping defined by $f\left(a_{i}\right)=\left[b_{i}\right)$ for $1 \leqq i \leqq n$. Then $f$ can be extended to a homomorphism $F: A \rightarrow S^{\prime}(B)$ by defining

$$
F(x)=\left\{\begin{array}{ccc}
\phi & \text { for all } & x \in A-\left[a_{1}\right) \\
{\left[b_{1}\right)} & \text { for all } & x \in\left[a_{1}\right)-\left[a_{2}\right) \\
\vdots & & \\
{\left[b_{n}\right)} & \text { for all } & x \in\left[a_{n}\right) .
\end{array}\right.
$$

It is a straightforward verification that $F$ is in $\mathscr{H}_{0}$. Thus the element $\prod_{i=1}^{n}\left(a_{i} \otimes b_{i}\right)$ uniquely determines the homomorphism $F$ given above. It is easy to verify that the subset $\left[\Pi_{i=1}^{n}\left(a_{i} \otimes b_{i}\right)\right]^{*}$ is isomorphic to $[F)=\left\{G \in \mathscr{H}_{0}: F \leqq G\right\}$, the principal upperhereditary subset of $\mathscr{H}_{0}$ determined by $F$. It is clear that any principal upper-hereditary subset of $\mathscr{H}_{0}$ is join-irreducible in the set of all such subsets of $\mathscr{H}_{0}$. Since $[F)$ is joinirreducible, the set representing $\Pi_{i=1}^{n}\left(a_{i} \otimes b_{i}\right)$ is join-irreducible in the ring of subsets isomorphic to $A \otimes B$ and so $\prod_{i=1}^{n}\left(a_{i} \otimes b_{i}\right)$ is join-irreducible in $A \otimes B$.

A partial converse to Theorem 2.2 holds. We will need the following lemma which is applicable to the homomorphisms of Theorem 2.1 .

Lemma 2.3. Let $A$ and $B$ be distributive lattices and let $f: A \rightarrow B$ be a homomorphism which is such that the homomorphic image of $A$ is a chain. Let $\theta$ be the congruence relation which is defined on $A$ by the homomorphism $f$. Then each non-empty congruence class in $A$ is uniquely determined as the intersection of a prime filter and a prime ideal.

Proof. Let $[a] \theta$ be a non-empty congruence class in $A$. It is well known that $[a] \theta$ can be uniquely determined as the intersection of an ideal $I$ in $A$ and a filter $D$ in $A$. We need only show that $I$ and $D$ are prime.

Suppose that $I$ is not prime. Then there exist $x_{1}, x_{2} \in A$ such that $x_{1}, x_{2} \notin I$ but $x_{1} x_{2} \in I$. Then $x_{1}+a$ and $x_{2}+a$ are not in $I$. But $\left(x_{1}+a\right)\left(x_{2}+a\right)=x_{1} x_{2}+x_{1} a+a x_{2}+a=$ $x_{1} x_{2}+a$ is in $I$ since $x_{1} x_{2} \in I$ and $a \in I$. Now $a \in D$ and $x_{1} x_{2}+a \geqq a$ so $x_{1} x_{2}+a$ is in 
$D$. Thus $x_{1} x_{2}+a \in I \cap D=[a] \theta$. But $x_{1}+a, x_{2}+a \notin[a] \theta$. Since $f$ is a homomorphism, $f\left(x_{1} x_{2}+a\right)=f\left(\left(x_{1}+a\right)\left(x_{2}+a\right)\right)=f\left(x_{1}+a\right) \cap f\left(x_{2}+a\right)$. The range of $f$ is a chain, so either $f\left(x_{1} x_{2}+a\right)=f\left(x_{1}+a\right)$ or $f\left(x_{1} x_{2}+a\right)=f\left(x_{2}+a\right)$. This contradicts the result that $x_{1} x_{2}+a \in[a] \theta$ and $x_{1}+a, x_{2}+a \notin[a] \theta$. Hence the ideal $I$ must be prime. A similar argument shows that $D$ is a prime filter.

We are now ready to present the following partial converse to Theorem 2.2. Our result requires the descending chain condition in $A$ and $B$ and includes the descending chain condition in $A \otimes B$ as a corollary. The lattice $L$ satisfies the descending chain condition if and only if every descending chain of elements of $L$ terminates at some point: if $a_{1} \geqq a_{2} \geqq \ldots \geqq a_{n} \geqq \ldots$ is any descending chain in $L$, there is some $k$ such that $a_{k}=a_{k+1}=\ldots$. If $L$ satisfies the descending chain condition, then every filter in $L$ is principal. In particular, every prime filter in $L$ is a principal filter that is generated by a join-irreducible element of $L$. The following theorem characterizes the join-irreducible elements of $A \otimes B$ when $A$ and $B$ satisfy the descending chain condition.

Theorem 2.4. Let $A$ and $B$ be distributive lattices in which the descending chain condition holds. Then each prime filter in $A \otimes B$ is principal and is generated by an element in $A \otimes B$ of the form $\prod_{i=1}^{n}\left(a_{i} \otimes b_{i}\right)$, where the sets $\left\{a_{i}\right\},\left\{b_{i}\right\}, 1 \leqq i \leqq n$, are sets of join-irreducibles in $A$ and $B$ such that $a_{1} \leqq \ldots \leqq a_{n}$ and $b_{1} \geqq \ldots \geqq b_{n}$.

Proof. We employ Lemma 2.3 to associate with each $F \in \mathscr{H}_{0}$, and thus with each $P \in S(A \otimes B)$, an element in $A \otimes B$ of the form $\prod_{i=1}^{n}\left(a_{i} \otimes b_{i}\right)$.

Let $F: A \rightarrow S^{\prime}(B), F \in \mathscr{H}_{0}$, be a homomorphism. Let $\theta$ be the congruence relation which is defined on $A$ by $F$ and let $[a] \theta$ be the congruence class of $\theta$ which is such that $F(x)=\phi$ for all $x \in[a] \theta$. Then $[a] \theta$ is a prime ideal in $A$ and the complement in $A$ of $[a] \theta$ is a prime filter. The descending chain condition in $A$ implies that this prime filter is principal. Let $a_{1}$ be the join-irreducible in $A$ which generates this prime filter. Then $F(x) \neq \phi$ for every $x \in A, x \geqq a_{1}$. Finally, $F\left(a_{1}\right)$ is a prime filter in $B$ and the descending chain condition in $B$ implies that this prime filter is principal. Let $b_{1}$ be the join-irreducible in $B$ which generates this prime filter. Then $F\left(a_{1}\right)=\left[b_{1}\right)$.

If $b_{1} \neq 0$, and if $A-\left(F^{-1}(\phi) \cup\left[a_{1}\right] \theta\right)$ is non-empty, we may then proceed analogously in determining the join-irreducibles $a_{2} \in A, b_{2} \in B$. Thus $a_{2}$ is the smallest $a \in\left[a_{1}\right)$ such that $F(a) \neq\left[b_{1}\right)$, and $b_{2}$ is the join-irreducible satisfying $F\left(a_{2}\right)=\left[b_{2}\right)$. The procedure is then repeated.

This construction is such that $a_{1} \leqq a_{2} \leqq \ldots$ and $b_{1} \geqq b_{2} \geqq \ldots$. The descending chain condition in $B$ ensures that the sequence of $b_{i}$ 's terminates at some point and that the above procedure ends after a finite number of applications.

We have thus associated with $F \in \mathscr{H}_{0}$ the uniquely determined pair of sequences $\left\{a_{i}\right\}$, $\left\{b_{i}\right\}, 1 \leqq i \leqq n$, of ascending and descending join-irreducibles in $A$ and $B$. By Theorem 2.2 we have that $\prod_{i=1}^{n}\left(a_{i} \otimes b_{i}\right)$ is join-irreducible in $A \otimes B$. The construction of the sequences $\left\{a_{i}\right\}$ and $\left\{b_{i}\right\}$ is such that $\bigcap_{i=1}^{n}\left\{G \in \mathscr{H}_{0}: b_{i} \in G\left(a_{i}\right)\right\}=[F)$, the principal upperhereditary subset of $\mathscr{H}_{0}$ generated by $F$. Hence $\bigcap_{i=1}^{n}\left(a_{i} \otimes b_{i}\right)^{*}$ is isomorphic to $[F)$, and $\Pi_{i=1}^{n}\left(a_{i} \otimes b_{i}\right)$ is a generator for the member of $S(A \otimes B)$ corresponding to the homomorphism $F$.

If $A$ and $B$ satisfy the descending chain condition, then Theorems 2.2 and 2.4 together characterize the join-irreducible elements of $A \otimes B$ as the elements of the form 
$\prod_{i=1}^{n}\left(a_{i} \otimes b_{i}\right)$, where $a_{i}$ and $b_{i}$ are join-irreducible in $A$ and $B$ and satisfy $a_{1} \leqq \ldots \leqq a_{n}$ and $b_{1} \geqq \ldots \geqq b_{n}$.

Corollary 2.5. Let $A$ and $B$ be distributive lattices in which the descending chain condition is satisfied. Then the following hold:

(i) Every filter in $A \otimes B$ is a principal filter.

(ii) The descending chain condition is satisfied in $A \otimes B$.

(iii) $A \otimes B$ is compactly generated by its join-irreducibles. (Thus, each element of $A \otimes B$ can be represented as a finite sum of join-irreducibles in $A \otimes B$.)

Proof. (ii) and (iii) are consequences of (i). (See Dilworth and Crawley [3], p. 70.) Theorem 2.4 showed that every prime filter in $A \otimes B$ is principal. It is well known that the lattice of all filters of a distributive lattice is compactly generated by the prime filters. It follows that every filter is principal.

If the descending chain condition is satisfied then Theorem 2.4 and Corollary 2.5(iii) provide us with a more straightforward procedure for constructing the tensor product than the method described in the remarks following Theorem 2.1. For a lattice $L$, let $J(L)$ denote the partially ordered set of non-zero join-irreducibles of $L$ and let $H(J(L))$ denote the lattice of lower-hereditary subsets of $J(L)$. If $L$ is a distributive lattice in which the descending chain condition holds, then $L$ and $H(J(L))$ are isomorphic ([6], p. 61). Now if $A$ and $B$ are distributive lattices satisfying the descending chain condition, then $A \otimes B$ also satisfies this condition. Hence in this case $A \otimes B$ is isomorphic to $H(J(A \otimes B))$. Theorem 2.4 provides a characterization of the elements of $J(A \otimes B)$, and Corollary 2.5 assures that the hereditary subset construction is applicable. Thus we can construct $A \otimes B$ by instead constructing $H(J(A \otimes B))$.

The following section is concerned with details and consequences of this construction.

\section{Applications and examples}

We begin with an illustration of the results of the preceding section. Let $A$ and $B$ be the lattices of Figure 1. Theorem 2.4 then permits us the construction of $J(A \otimes B)$ which is shown in Figure 2.

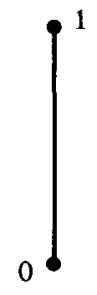

A

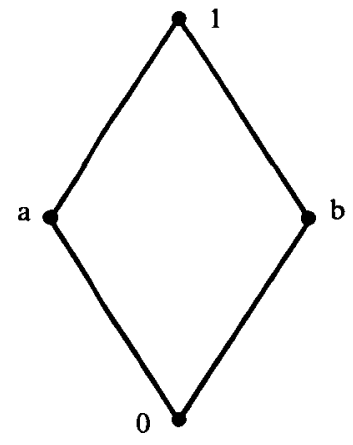

B

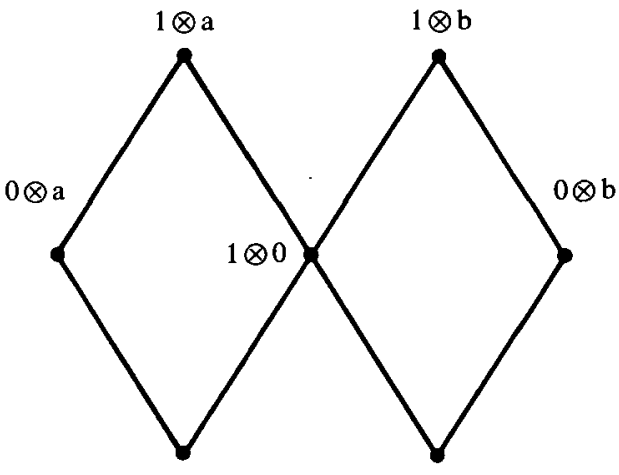

$(0 \otimes a)(1 \otimes 0)$
$J(A \otimes B)$

Figure 1

Figure 2 
We note, as an example of the sort of arguments which might return us to the partially ordered set $\mathscr{H}_{0}$ from $J(A \otimes B)$, that the homomorphism corresponding to the join-irreducible $(0 \otimes a)(1 \otimes 0)$ is the homomorphism $F: A \rightarrow S^{\prime}(B)$ defined by $F(0)=\{a, 1\}$ and $F(1)=B$. This homomorphism, in turn, corresponds to the bihomomorphism $g: A \times B \rightarrow 2$ defined by

$$
g(x, y)= \begin{cases}0 & \text { if }(x, y)=(0,0) \text { or }(0, b) \\ 1 & \text { otherwise }\end{cases}
$$

By determining the lattice of lower-hereditary subsets of $J(A \otimes B)$, we find that $H(J(A \otimes B))$, and hence $A \otimes B$ itself, is the 18-element distributive lattice given in Figure 3.

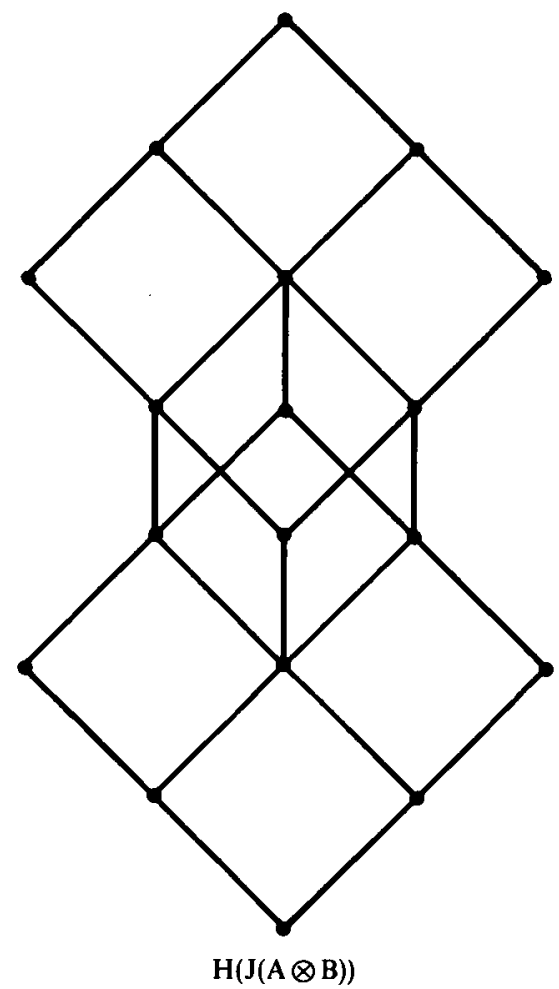

Figure 3

The general problem of identifying the join-irreducible products of degree $k$ in $A \otimes B$, by Theorem 2.4, reduces to that of identifying the distinct chains of exactly $k$ joinirreducibles in $A$ and $B$. This provides what appears to us to be the most efficient procedure for the enumeration of the members of $J(A \otimes B)$. Its application in the case that $A$ and $B$ are Boolean algebras is particularly simple.

The length of a finite distributive lattice $L$ is defined as the (uniquely determined) length of the maximal chains in $L$ and is equal to $|J(L)|$, the number of non-zero joinirreducibles in $L$. 
Theorem 3.1. Let $A$ and $B$ be the Boolean algebras on $m$ and $n$ generators respectively. Then the length of $A \otimes B$ is $m+2 m n+n$ and $A \otimes B$ is a subdirect product of $\max \{m n, m+n\}$ chains.

Proof. Let $A$ be the Boolean algebra with generators $\left\{a_{1}, \ldots, a_{m}\right\}$ and $B$ be the Boolean algebra with generators $\left\{b_{1}, \ldots, b_{n}\right\}$. Then the non-zero join-irreducibles in $A \otimes B$ are the $m n$ products of the form $\left(a_{i} \otimes 0\right)\left(0 \otimes b_{j}\right), 1 \leqq i \leqq m, 1 \leqq j \leqq n$, the $m+n$ join-irreducible generators of the form $\left(a_{i} \otimes 0\right)$ or $\left(0 \otimes b_{j}\right)$, and the $m n$ joinirreducible generators of the form $\left(a_{i} \otimes b_{j}\right), 1 \leqq i \leqq m, 1 \leqq j \leqq n$. Thus $A \otimes B$ has a total of $m n+m+n+m n=m+2 m n+n$ non-zero join-irreducible elements. Hence the length of $A \otimes B$ is $m+2 m n+n$.

The covering relations in $J(A \otimes B)$ may be completely described with reference to the join-irreducibles of the form $\left(a_{i} \otimes 0\right)$ or $\left(0 \otimes b_{j}\right)$. The join-irreducibles $\left(a_{i} \otimes 0\right)$ cover each product $\left(a_{i} \otimes 0\right)\left(0 \otimes b_{j}\right), 1 \leqq j \leqq n$, and are covered by each generator $\left(a_{i} \otimes b_{j}\right)$, $1 \leqq j \leqq n$. Analogous statements hold for elements of the form $\left(0 \otimes b_{j}\right)$. Hence there are $m+n$ elements which cover $m n$ elements and are covered by $m n$ elements.

The preceding enumeration of the members and the covering relations in $J(A \otimes B)$ implies that the largest anti-chain in $J(A \otimes B)$ is of cardinality $\max \{m n, m+n\}$. We may then apply Dilworth's decomposition theorem and its consequences (see [2]) to argue that there is a representation, not necessarily unique, of $A \otimes B$ as a subdirect product of $\max \{m n, m+n\}$ chains.

The construction which opened this section provides an illustration of the above. One further result which is illustrated by the lattices of Figure 1 concerns projectivity in the tensor product.

Theorem 3.2. The tensor product of a finite projective distributive lattice and a finite chain is projective.

Proof. For a lattice $L$, let $J_{01}(L)$ denote the set $J(L) \cup\{0,1\}$. Balbes has shown that if $L$ is a finite distributive lattice, then $L$ is projective if and only if the partially ordered set $J_{01}(L)$ is a lattice ([1], Theorem 7.4). For distributive lattices $A$ and $B$, let $\operatorname{Hom}(A, B)$ denote the partially ordered set of lattice homomorphisms from $A$ into $B$. A result of Kucera and Sands asserts that if $A$ is a projective distributive lattice, then $\operatorname{Hom}(A, B)$ is a lattice $([7]$, Theorem 4.1).

Now assume that $A$ is a finite projective distributive lattice and $B$ is a finite chain. Then $S^{\prime}(B)$ is also a finite chain. It follows from Theorem 2.1 that $S^{\prime}(A \otimes B)$ is isomorphic to $\operatorname{Hom}\left(A, S^{\prime}(B)\right)$. By the theorem of Kucera and Sands, $\operatorname{Hom}\left(A, S^{\prime}(B)\right)$ is a lattice, and so $S^{\prime}(A \otimes B)$ is also a lattice. Now $J_{01}(A \otimes B)$ is isomorphic to the dual of $S^{\prime}(A \otimes B)$. Hence $J_{01}(A \otimes B)$ is a lattice, and it follows from the result of Balbes that $A \otimes B$ is projective.

\section{REFERENCES}

1. R. Balbes, Projective and injective distributive lattices, Pacific J. Math. 21 (1967), 405-420.

2. R. P. Dilworth, A decomposition theorem for partially ordered sets, Annals of Math. 51 (1950), 161-166. 
3. R. P. Dilworth and P. Crawley, Algebraic Theory of Lattices (Prentice-Hall, Englewood Cliffs, N.J., 1973).

4. G. A. Fraser, The tensor product of distributive lattices, Proc. Edinburgh Math. Soc. 20 (1976), 121-131.

5. G. A. Fraser, The tensor product of distributive lattices II, Proc. Edinburgh Math. Soc. 20 (1977), 355-360.

6. G. Grätzer, General Lattice Theory (Academic Press, New York, 1978).

7. T. G. KuCERA and B. SANDs, Lattices of lattice homomorphisms, Algebra Universalis 8 (1978), 180-190.

UNIVERSITY OF ILLINOIS

URBANA, ILLINOIS 61801

California State University

Los Angeles, California 90032
California State University

Hayward, California 94542 